\title{
Relating Rapid Chloride Migration Coefficient of Blast Furnace Slag Concrete to Capillary Pore Structure Parameters
}

\author{
Chao Yang ${ }^{1}$, Shuguang $\mathrm{Wang}^{1}$, Feng $\mathrm{Xu}^{1}$, Weiwei $\mathrm{Li}^{1}$, and Dongsheng $\mathrm{Du}^{1}$ \\ ${ }^{1}$ College of Civil Engineering, Nanjing Tech University, Nanjing, 211816, P. R. China
}

\begin{abstract}
Blast furnace slag blended concrete is widely used in infrastructure, and its chloride resistance is of great concern. This paper experimentally investigated the capillary pore structure and chloride resistance of blast furnace slag blended concrete. Blast furnace slag was proved to be able to optimize the critical pore radius and decrease the proportion of detrimental capillary pores (with radius between $50 \mathrm{~nm}$ and 10,000 nm). Meanwhile, the benefit of BFS in improving the chloride resistance was proved. Finally, regression analysis showed that the rapid migration coefficient is proportional to the critical pore radius and the detrimental capillary pore proportion. Nevertheless, the rapid migration coefficient is not closely related to the capillary porosity.
\end{abstract}

\section{Introduction}

The chloride induced corrosion of reinforcement has been a severe durability problem for reinforced concrete $(\mathrm{RC})$ members for decades [1,2]. This situation is becoming worse since more and more RC infrastructures will reach the designed service life in the next few decades, especially in industrialized countries [3]. Therefore, various mineral replacement materials such as fly ash and blast furnace slag are used in the concrete mixture to improve the chloride resistance of concrete.

Blast furnace slag (BFS) is a byproduct of the iron production and has a cementitious nature. It is known that BFS can optimize the pore structure and improve the chloride resistance of concrete because of the pozzolanic reaction product. The modification of pore structure of BFS concrete is assumed to be the main reason why BFS concrete exhibits a good chloride resistance[4]. However, it has also been pointed out that only capillary pores in some ranges can affect the chloride resistance $[5,6]$. Therefore, it is necessary to investigate which capillary pore structure characteristics can actually affect the chloride resistance of BFS concrete.

This paper presents an attempt to relate the rapid chloride migration coefficient to the capillary pore structure characteristics such as capillary porosity, critical pore radius and detrimental capillary pore proportion for BFS concrete with different water to binder (W/B) ratio and curing time. In this paper, two different $\mathrm{W} / \mathrm{B}$ ratio (0.35 and 0.43) and two different curing time (28 and 90 days) were employed for concrete specimens. The capillary pore structure was measured by the mercury intrusion porosimetry (MIP) test, and the chloride resistance was tested by the rapid chloride migration coefficient (MIP) test. The influence of BFS dosage, W/B ratio and curing time on the capillary pore structure and chloride resistance was investigated based on the experimental results. It was found that the chloride resistance of BFS concrete was proportional to the critical pore radius and detrimental capillary pore proportion after the linear regression analysis.

\section{Experimental procedure}

\subsection{Materials and specimens}

Materials employed for the casting of concrete specimens include Portland cement, coarse aggregate, fine aggregate and blast furnace slag. The cement was Type I Portland cement concrete conforming to ASTM C150 with a compressive strength of $52.5 \mathrm{~N} / \mathrm{mm} 2$. For aggregate, crushed limestone was used as coarse aggregate and river sand was used as fine aggregate. The blast furnace slag used was type S95 according to the Chinese National Standard GB/T203.

The concrete specimens can be classified into two groups with different target compressive strengths: 35 $\mathrm{MPa}$ and $55 \mathrm{MPa}$. For each group, four different proportions of blast furnace slag were added into the 
mixture, which were $0 \%, 20 \%, 30 \%$ and $40 \%$, respectively. Detailed mixture proportions are listed in Table 1. All concrete specimens were cylinders (diameter
$100 \mathrm{~mm} \times$ height $200 \mathrm{~mm}$ ) and were cured until the target ages (28 and 90 days).

Table 1. Mix proportions of concrete.

\begin{tabular}{|c|c|c|c|c|c|c|c|c|}
\hline \multirow[b]{2}{*}{ Sample ID } & \multirow[b]{2}{*}{$\mathrm{W} / \mathrm{B}$} & \multirow{2}{*}{$\begin{array}{c}\text { Blast Furnace Slag } \\
\text { dosage }(\%)\end{array}$} & \multicolumn{6}{|c|}{ Unit content $(\mathrm{kg} / \mathrm{m} 3)$} \\
\hline & & & Water & Cement & Blast Furnace Slag & Fine aggregate & $\begin{array}{c}\text { Coarse } \\
\text { aggregate }\end{array}$ & SP \\
\hline $\mathrm{C} 1$ & 0.43 & 0 & 160 & 370 & 0 & 770 & 1030 & 2.6 \\
\hline $\mathrm{C} 2$ & 0.43 & 20 & 160 & 296 & 74 & 770 & 1030 & 2.6 \\
\hline $\mathrm{C} 3$ & 0.43 & 30 & 160 & 259 & 111 & 770 & 1030 & 2.6 \\
\hline $\mathrm{C} 4$ & 0.43 & 40 & 160 & 222 & 148 & 770 & 1030 & 2.6 \\
\hline $\mathrm{C} 5$ & 0.35 & 0 & 154 & 440 & 0 & 685 & 1120 & 3.97 \\
\hline C6 & 0.35 & 20 & 154 & 352 & 88 & 685 & 1120 & 3.97 \\
\hline $\mathrm{C} 7$ & 0.35 & 30 & 154 & 308 & 132 & 685 & 1120 & 3.97 \\
\hline $\mathrm{C} 8$ & 0.35 & 40 & 154 & 264 & 176 & 685 & 1120 & 3.97 \\
\hline
\end{tabular}

In this research, the chloride resistance of concrete was measured by the rapid chloride migration (RCM) test, and the capillary pore structure of concrete was measured by the mercury intrusion porosimetry (MIP) test. Therefore, the concrete cylinders were further handled before these two tests. For the RCM test, the 50mm-thick cylindrical specimens were sliced out of the concrete cylinders and were saturated in the $\mathrm{Ca}(\mathrm{OH})_{2}$ solution for 18 hours. For the MIP test, the cylinders were crumbed into small fragments around $0.1 \mathrm{~cm}^{3}$.

\subsection{Test methods}

\subsubsection{Rapid chloride migration test (RCM)}

The chloride resistance of concrete specimens were measured by the RCM test conforming to NT Build 492 [7] at 28 or 90 days. The chloride ion ingress was accelerated by the current. After that, the final chloride penetration depth was measured by the spray of $\mathrm{AgNO}_{3}$ solution. Finally, the RCM coefficient was calculated based on the penetration depth.

\subsubsection{Mercury intrusion porosimetry (MIP)}

The capillary pore structure of concrete specimens was measured by the MIP test on the Poremaster GT-60 mercury porosimeter at 28 or 90 days. Herein, the capillary pores refer to those pores with radius ranging from $10 \mathrm{~nm}$ to $10,000 \mathrm{~nm}$ according to Mindess et al. [8]. The capillary porosity, the critical pore radius and the distribution of the capillary pores were extracted from the data in tests.

\section{Results}

\subsection{Capillary pore structure parameters of BFS blended concrete}

\subsubsection{Capillary porosity}

The capillary porosity of all concrete specimens is plotted in Figure 1 against the dosage of blast furnace slag. From Figure 1, it can be observed that the capillary porosity of concrete specimens with the $\mathrm{W} / \mathrm{B}$ ratio of 0.43 is larger than that with the $\mathrm{W} / \mathrm{B}$ ratio of 0.35 . It implies that the $\mathrm{W} / \mathrm{B}$ ratio is a predominant factor that will influence the development of capillary pore structure for BFS concrete. It coincides with the conclusion for the ordinary concrete. However, the correlation between the capillary porosity and the dosage of BFS is slightly complex. Specifically, at 28 days, the capillary porosity of BFS concrete is increased with the dosage of BFS. Nevertheless, at 90 days, the capillary porosity is no longer monotonically related to the proportion of BFS. This means that the addition of BFS cannot optimize the capillary porosity and even have detrimental influence.

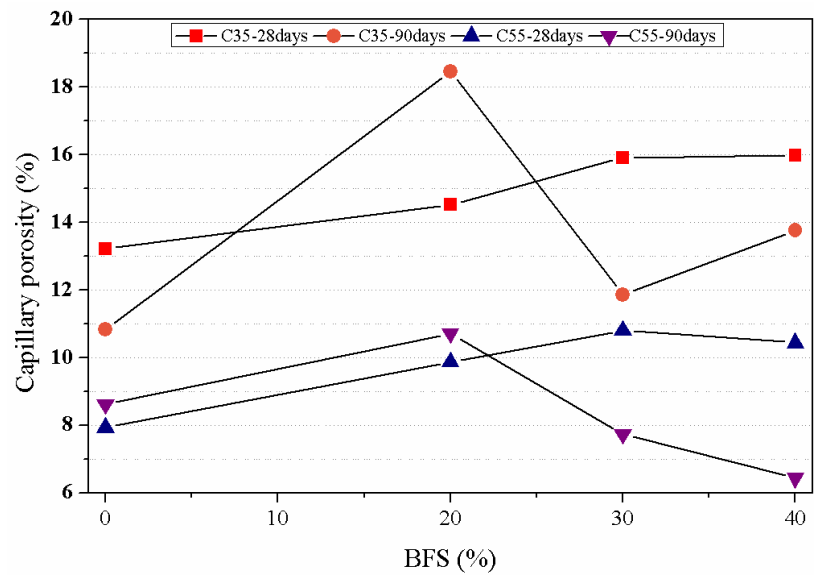

Figure 1. The capillary porosity of concrete specimens at 28 and 90 days. 


\subsubsection{Critical pore radius}

The critical pore radius of all concrete specimens at 28 and 90 days is plotted in Figure 2. Generally, the critical pore radius is increased with the proportion of BFS, which means that a finer capillary pore structure is obtained because of the addition of BFS. This phenomenon agrees with that in Uysal [9] whose research has proved that BFS can lead to a denser and discontinuous pore system. This should be due to the fact that the pozzolanic reaction product of BFS can fill the pores and decrease the connectivity of pore system. Note that the critical pore radius of concrete specimens (W/B ratio $=0.35$ ) at 28 days is almost constant. However, it doesn't mean that BFS does not work at this time since BFS is highly cementitious [10]. This phenomenon should be a coincidence.

Moreover, the critical pore radius of nearly all concrete specimens at 90 days is smaller than that at 28 days. It is in agreement with the common sense. A longer curing time allows a more complete BFS pazzolanic reaction and cement hydration, making more reaction product fill the initial pores and ensuring a discontinuous pore system.

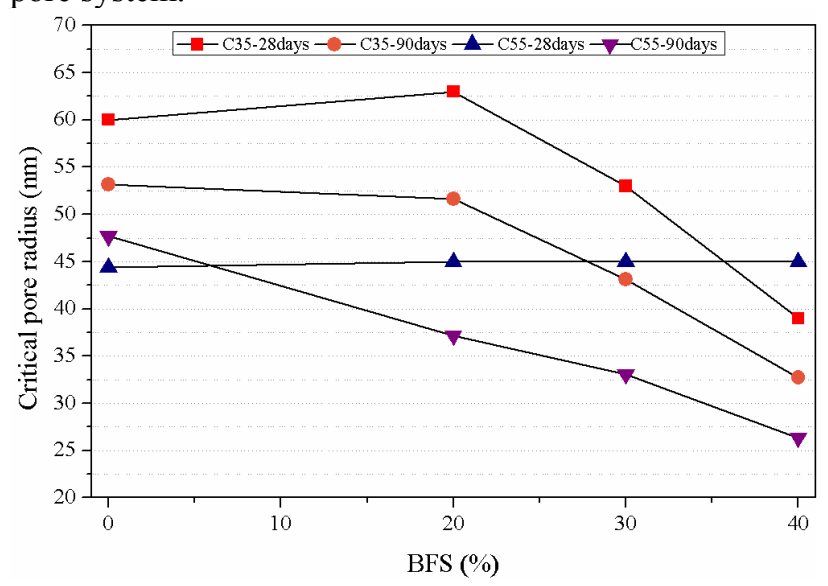

Figure 2. The critical pore radius of concrete specimens at 28 and 90 days.

\subsubsection{Capillary pore size distribution}

To have a better understanding of the influence of BFS on the capillary pore structure of concrete, the capillary pore system needs to be subdivided. Kropp [5] found that only capillary pore larger than $50 \mathrm{~nm}$ can affect the permeability of concrete. Therefore, in this paper, these capillary pores larger than $50 \mathrm{~nm}$ are defined as detrimental capillary pores, and the rest capillary pores are assumed to be normal capillary pores. The percentage of detrimental capillary pore among the whole capillary pore system is a good parameter that can represent the quality of the capillary pore system and is named as "detrimental capillary pore proportion". The detrimental capillary pore proportion at 28 and 90 days is presented in Figure 3.

The general trend is that the detrimental capillary pore proportion descends with the increase of BFS dosage. When the $\mathrm{W} / \mathrm{B}$ ratio is 0.43 , the detrimental capillary pore proportion of concrete specimens $(40 \% \mathrm{BFS})$ is
$36.87 \%$ and $38.46 \%$ of the ordinary concrete at 28 and 90 days, respectively. When the $\mathrm{W} / \mathrm{B}$ ratio is 0.35 , the corresponding value is $54.12 \%$ and $11.23 \%$, respectively. It means that the quality of capillary pore system is optimized with the presence of BFS, and the effectiveness of BFS increases with the dosage of BFS. Moreover, it can be observed that the detrimental capillary pore proportion at 90 days is smaller than that at 28 days, which implies that the quality of capillary pore structure is also affected by the curing time.

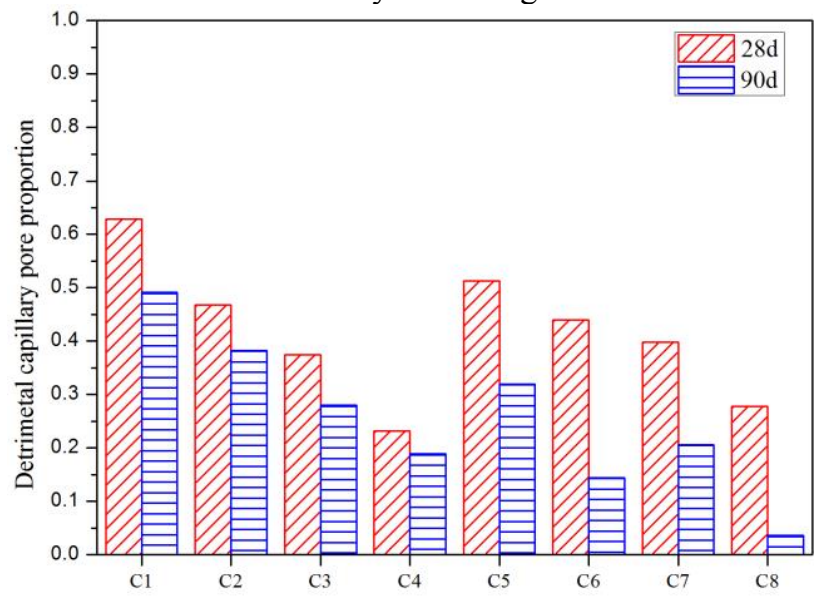

Figure 3. The detrimental capillary pore proportion of concrete specimens at 28 and 90 days.

\subsection{The rapid chloride migration coefficient}

The RCM coefficient of the concrete specimens at 28 days and 90 days is shown in Figure 4. It can be seen that the RCM coefficient is decreased with the increase of BFS dosage. For example, the RCM coefficients of the concrete specimens $(\mathrm{W} / \mathrm{B}$ ratio $=0.43)$ at 28 days blended with $20 \%, 30 \%$ and $40 \%$ of BFS are $80.98 \%, 65.56 \%$ and $61.76 \%$ of that of ordinary concrete specimens. This should result from the pozzolanic reaction of BFS. On one hand, the pozzolanic reaction product fill the initial pores, resulting in a denser pore structure. On the other hand, more Friedel's salt is produced and the chloride binding capacity is improved. Since the presence of BFS improves both the physical and chemical aspects of concrete, the chloride resistance is significantly improved, which is represented by a lower RCM coefficient.

With regard to the influence of W/B ratio and curing time, the following tendency can be observed: (1) a longer curing time leads to a lower RCM coefficient; (2) a lower W/B ratio results in a lower RCM coefficient. These two conclusions are not hard to understand and have been acknowledged in the field of ordinary concrete. Similar to the hydration of cement, the pozzolanic reaction of BFS also requires enough time. Therefore, it can be deduced that the RCM coefficient will be lower at later ages. It is notable that although more water can accelerate the reaction of BFS to help optimize the pore structure so as to strengthen the chloride resistance to some extent, the initial capillary pore structure has been degraded resulting from the high W/B ratio. That is to say: $\mathrm{W} / \mathrm{B}$ ratio seems to be a very important parameter when considering the chloride resistance of BFS concrete and 
might be more important than the dosage of BFS. For example, at 28 days, the RCM coefficient of C6 (W/B ratio $=0.35,20 \% \mathrm{BFS})$ is $89.08 \%$ of that of $\mathrm{C} 4(\mathrm{~W} / \mathrm{B}$ ratio $=0.43,50 \% \mathrm{BFS}$ ).

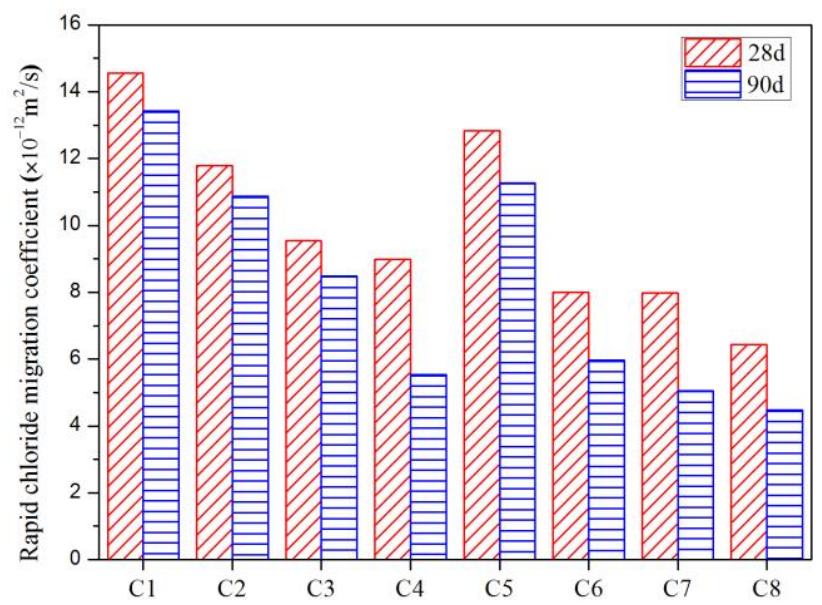

Figure 4. The RCM coefficients of concrete specimens at 28 and 90 days.

\section{Further analysis}

Above has given some simple analysis about the results obtained from experiments, and some qualitative conclusions have been drawn about the capillary pore structure and chloride resistance of BFS concrete under different circumstance. Normally, people tends to predict chloride resistance of BFS concrete based on parameters such as W/B ratio, curing time, BFS dosage. However, the chemical and physical characteristics of raw materials might be different, making this type of prediction unreliable. To ensure a more reliable prediction of chloride resistance of BFS concrete, it would be better to predict chloride resistance based on the capillary pore structure. Thus, in this section, the capillary pore structure parameters are plotted against the RCM coefficient to see whether there is any correlation.

\subsection{Capillary porosity and RCM coefficient}

The RCM coefficients of BFS concrete specimens are plotted in Figure 5 against the capillary porosities. The data points in the figure are scattered, and no trend can be observed. This indicates that the influence of capillary porosity on chloride resistance of BFS concrete is quite limited. Although this conclusion seems to be different from the common sense that concrete with a high porosity should have a bad chloride resistance, it is reasonable as only those "effective" capillary pores can allow the ingress of chloride. And, those "effective" capillary pores are the detrimental capillary pores defined before. Therefore, not only the common practice to relate the chloride resistance of concrete to the porosity is not reasonable but relating the chloride resistance to the capillary porosity is also unreliable.

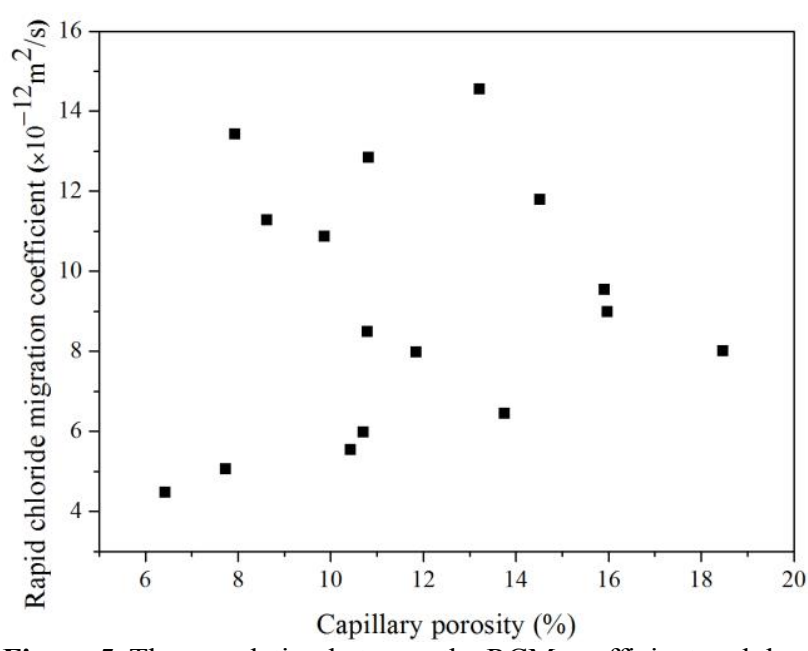

Figure 5. The correlation between the RCM coefficient and the capillary porosity.

\subsection{Critical pore radius and RCM coefficient}

The RCM coefficients of BFS concrete specimens are plotted in Figure 6 against the critical pore radius. Generally, it can be seen that the RCM coefficient increases with the critical pore radius. The critical pore radius is a parameter that represents the connectivity of pore structure, and a large value of critical pore radius means a continuous pore system [11]. It is known that a continuous system is prone to be invaded by chloride ion. Theoretically, the phenomenon observed is credible.

Moreover, a linear trend can be observed among the data points. The linear regression analysis shows that the coefficient of determination $\left(\mathrm{R}^{2}\right)$ is 0.6727 , which indicates that the linear correlation between the critical pore radius and the RCM coefficient is relatively strong. Therefore, it can be concluded that critical pore radius is a very important parameter related to the chloride resistance and should be considered in the future work about the prediction of RCM coefficient.

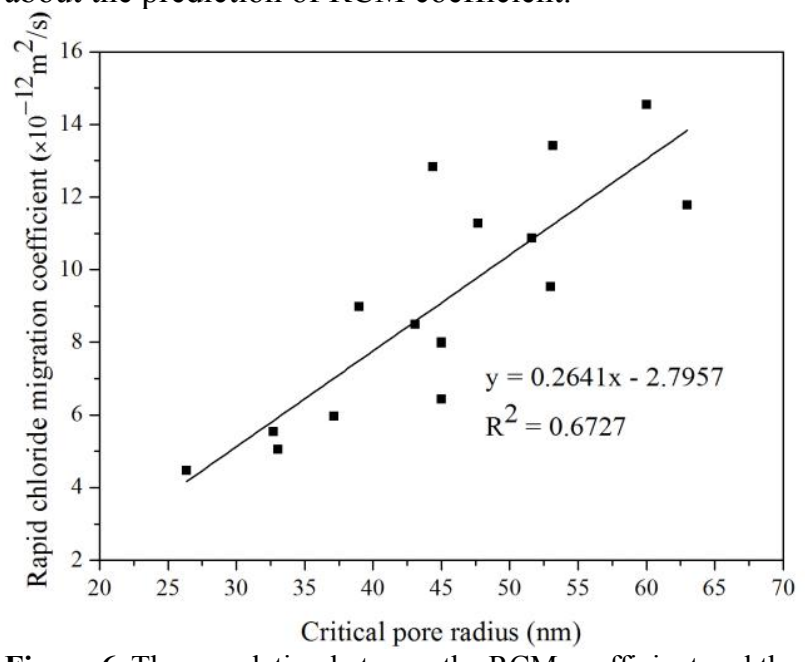

Figure 6. The correlation between the RCM coefficient and the critical pore radius. 


\subsection{Detrimental capillary pore proportion and RCM coefficient}

Figure 7 plots the RCM coefficient of BFS concrete specimens against the detrimental capillary pore proportion. In the figure, the RCM coefficient increases with the detrimental capillary pore proportion. Since the detrimental capillary pore proportion represents the quality of the capillary pore structure, the figure indicates that a good quality of capillary pore structure is beneficial to the chloride resistance of BFS concrete. It is in agreement with the conclusion drawn by Yang [12] to some extent.

To investigate the correlation between RCM coefficient and detrimental capillary pore proportion quantitatively, the linear regression analysis is done here. The coefficient of determination $\left(\mathrm{R}^{2}\right)$ is 0.75478 , which implies that the RCM coefficient should be linearly related to the detrimental capillary pore proportion. It also proves that not all the capillary pore can affect the chloride ingress for BFS concrete. Moreover, it can be deduced that the prediction equation of RCM coefficient should include the detrimental capillary pore proportion.

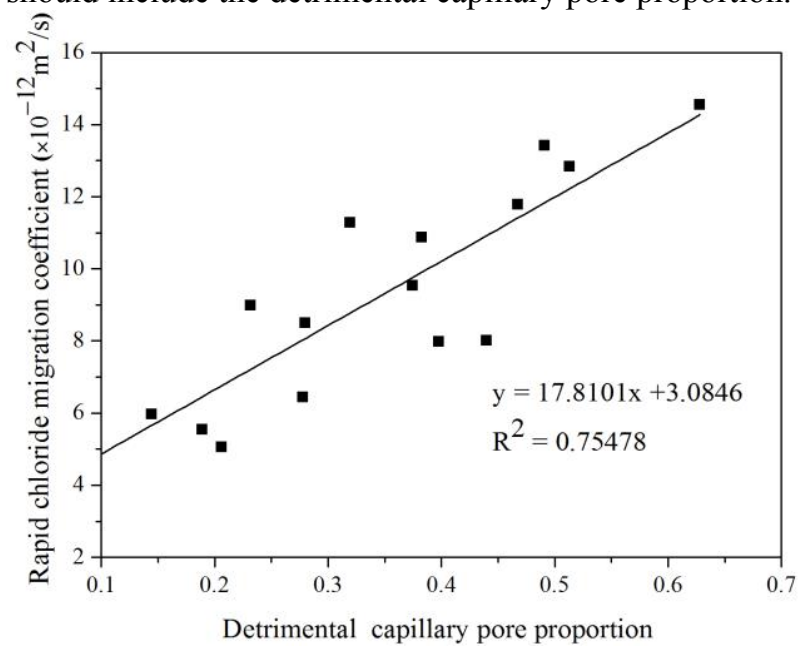

Figure 7. The correlation between the RCM coefficient and the detrimental capillary pore proportion.

\section{Conclusions}

Based on the experimental results and the discussion above, the following conclusions can be drawn:

(1) The presence of BFS can optimize the capillary pore structure of BFS concrete through reducing the connectivity of capillary pore structure and decreasing the detrimental capillary pore proportion. However, the influence of BFS on the capillary porosity is quite limited. Moreover, BFS is very effective in improving the chloride resistance of BFS concrete.

(2) Both W/B ratio and curing time can affect the critical pore radius and the detrimental capillary pore proportion. A lower $\mathrm{W} / \mathrm{B}$ ratio and a longer curing time is more beneficial. With respect to the chloride resistance of BFS concrete, a low W/B ratio and a long curing time is also effective.
(3) The capillary porosity does not have significant influence on the chloride resistance of BFS concrete. However, both the critical pore radius and the detrimental capillary pore proportion are linearly correlated with the RCM coefficient of BFS concrete. The RCM coefficient increases with the critical pore radius and the detrimental capillary pore proportion. It is deduced that the critical pore radius and the detrimental capillary pore proportion should be the main parameters included in the prediction formula of the RCM coefficient of BFS concrete.

\section{References}

1. C.L. Page, Mechanism of corrosion protection in reinforced concrete marine structures, Nature. $\mathbf{2 5 8}$ (1975)

2. C.L. Page, M.M. Page, Durability of concrete and cement composites, (Woodhead Publishing Ltd, 2007)

3. U.M. Angst, B. Elsener, Sci. Adv. 3 (2017)

4. E. Özbay, M. Erdemir, H.I. Durmuş, Constr. Build. Mater. 105 (2016)

5. J. Kropp, H.K. Hilsdorf, Performance Criteria for concrete Durability: RILEM Report 12, Penetration Resist. (1995)

6. F. Massazza, Lea's Chemistry of Cement and Concrete (Fourth Edition), 10 (2003)

7. NT Build 492, Concrete, mortar and cement-based repair materials: Chloride migration coefficient from non-steady-state migration experiments, Nord. Method. (1999)

8. S. Mindess, J.F. Young, D. Darwin, Concrete, (Prentice Hall, 2003)

9. M. Uysal, K. Yilmaz, M. Ipek, Constr. Build. Mater. 27 (2012)

10. X. Shi, N. Xie, K. Fortune, J. Gong, Constr. Build. Mater. 30 (2012)

11. N. Neithalath, J. Jain, Cem. Concr. Res. 40 (2010)

12. C.C. Yang, S.W. Cho, L.C. Wang, Mater. Chem. Phys. 100 (2006) 